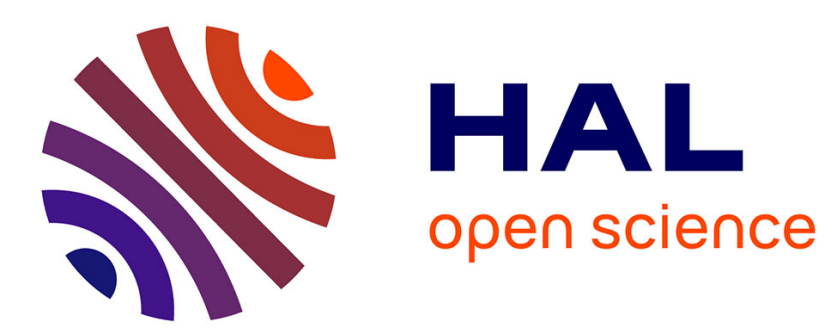

\title{
Optimal control for rough differential equations
}

Laurent Mazliak, Ivan Nourdin

\section{- To cite this version:}

Laurent Mazliak, Ivan Nourdin. Optimal control for rough differential equations. 2006. hal-00077917

\section{HAL Id: hal-00077917 \\ https://hal.science/hal-00077917}

Preprint submitted on 1 Jun 2006

HAL is a multi-disciplinary open access archive for the deposit and dissemination of scientific research documents, whether they are published or not. The documents may come from teaching and research institutions in France or abroad, or from public or private research centers.
L'archive ouverte pluridisciplinaire HAL, est destinée au dépôt et à la diffusion de documents scientifiques de niveau recherche, publiés ou non, émanant des établissements d'enseignement et de recherche français ou étrangers, des laboratoires publics ou privés. 


\title{
Optimal control for rough differential equations
}

\author{
Laurent Mazliak* and Ivan Nourdin \\ Université Pierre et Marie Curie (Paris 6) \\ Laboratoire Probabilités et Modèles Aléatoires (LPMA) \\ Boite courrier 188, F-75252 Paris Cedex 5 \\ \{mazliak, nourdin\}@ccr.jussieu.fr
}

\begin{abstract}
In this note, we consider an optimal control problem associated to a differential equation driven by a Hölder continuous function $g$ of index $\beta>1 / 2$. We split our study in two cases. If the coefficient of $d g_{t}$ does not depend on the control process, we prove an existence theorem for a slightly generalized control problem, that is we obtain a literal extension of the corresponding deterministic situation. If the coefficient of $d g_{t}$ depends on the control process, we also prove an existence theorem but we are here obliged to restrict the set of controls to sufficiently regular functions.
\end{abstract}

Key words: Optimal control - Rough differential equations - Fractional Brownian motion - Young integral - Doss-Sussmann's method.

\section{Introduction}

In recent years, several authors have been interested in control problems involving a stochastic process driven by a fractional Brownian motion (fBm in short). This kind of situation may indeed be natural when one wishes to modelized a problem in which long distance memory effects may occur. However, contrary to the situation

${ }^{*}$ Corresponding author. Fax: +331 47277223 
of processes driven by ordinary Brownian motion, fBm lacks many strong stochastic properties (in particular, martingales properties). Although attempts have been made to build a stochastic calculus for fBm some twenty years ago (see [1] for a nice survey), the developed techniques remain until today not so easy to use.

In order to deal with the situation of control for processes driven with $\mathrm{fBm}$, it was up to now necessary to limit oneself to special situations. Two directions have been mainly considered in recent literature:

1. A series of paper used a stochastic calculus based on Wick integrals and developed in [3]; see in particular [9] for considerations about application to mathematical finance. Moreover, in a recent paper [10], the special situation of a linear regulator driven by fBm was considered and optimal controls characterized.

2. A completely different way has been followed in several papers of a group in France, but only when the considered problem is strongly linear, a situation permitting to the author to use a martingale representation of $\mathrm{fBm}$ in order to transform the original problem in an ordinary stochastic control problem (see in particular [11] for a general exposition of the method).

In the present paper, we also keep a stochastic control problem for a process driven by a fBm in the background, and we want to study existence results for optimal controls. Nevertheless, as the basic property we need in our approach occurs to be the Hölder regularity of the paths of $\mathrm{fBm}$, we study here the deterministic situation where the state under control is driven by a Hölder continuous function $g$ of index $\beta$

$$
x_{t}^{u}=x_{0}^{u}+\int_{0}^{t} \sigma\left(s, u_{s}, x_{s}^{u}\right) d g_{s}+\int_{0}^{t} b\left(s, u_{s}, x_{s}^{u}\right) d s, \quad t \in[0, T],
$$

where the control process $u$ belongs to a set of admissible controls $\mathcal{U}$. When $\beta \in$ $(1 / 2,1)$, it is possible to choose Young integral [17] for integration with respect to $d g_{t}$ in (11), which simply appears to be the limit of Riemann sums. Moreover, as it was remarked in [19], it is possible to express it in terms of fractional derivative operators (see Section 2 below). This appears to be very useful to allow easier computations, and mostly has motivated us to restrict to the case where $\beta \in(1 / 2,1)$ in order to choose the Young integral. The next step would be considering the case where $\beta \in(1 / 3,1 / 2]$. In the very recent work [ 8$]$, an equivalent to the expression of the Young integral in terms of fractional derivative operators has been proposed, using quadratic multiplicative functional. Thus, the strategy introduced in this paper could also certainly be derived for $\beta \in(1 / 3,1 / 2]$ and we propose to study this fact in a forthcoming paper. 
The control problem considered in the present paper can be set in the following way.

Problem: 'A cost functional $J: \mathcal{U} \rightarrow \mathbb{R}$ being given, is it possible to prove the existence of $u^{*} \in \mathcal{U}$ realizing $\inf _{u \in \mathcal{U}} J(u)$ ?'

As usual, the bigger $\mathcal{U}$ is, the more difficult it is to answer this question. A general methodology is to look for conditions ensuring that $\mathcal{U}$ be compact for a certain topology under which $J$ is continuous.

Differential equations of the type (1) (without the control process $u$ ) have been intensively studied in recent years, due in particular to possible applications for fBm (see, e.g., [7, 8, 13, 14, 16]). Since the obtention of solutions to (11) requires in general regularity on the coefficients (see Theorem 3 below), we split our study in two cases.

1. If the coefficient of $d g_{t}$ does not depend on the control process, we are able to extend the deterministic situation, and to prove an existence theorem for a slightly generalized control problem where the controls are in fact randomized : see Corollary 1 and Proposition [4. In fact, we use the so-called 'compactification methods', which have been developed during the 1960's for deterministic control problems (see [6], [18]), and during the 1970's for the stochastic control problem (see [5], [四).

2. If the coefficient of $d g_{t}$ does depend on the control function, the situation is much more intricate, and this obliges us to severely restrict the set of controls to sufficiently regular functions. A challenging question would be to relax this hypothesis, but this would require to get a reasonable notion of solution for a differential equation with a weaker regularity than Hölderian. This seems not to be already available in the literature, up to our best knowledge.

The paper is organized as follows. In section 2 , we recall some now classical facts on fractional integrals and derivatives, which are useful for the sequel. In section 3 , we study the optimal control problem in the case where $\sigma$ does not depend on $u$. The case where $\sigma$ depends on $u$ is considered in section 4 .

\section{Fractional integrals and derivatives}

Let $a, b \in \mathbb{R}, a<b$. For any $p \geq 1$, we denote by $L^{p}=L^{p}([a, b])$ the usual Lebesgue spaces of functions on $[a, b]$.

Let $f \in L^{1}$ and $a>0$. The left-sided and right-sided fractional RiemannLiouville integrals of $f$ of order $\alpha$ are defined for almost all $x \in(a, b)$ by

$$
I_{a+}^{\alpha} f(x)=\frac{1}{\Gamma(\alpha)} \int_{a}^{x}(x-y)^{\alpha-1} f(y) d y
$$


and

$$
I_{b-}^{\alpha} f(x)=\frac{(-1)^{-\alpha}}{\Gamma(\alpha)} \int_{x}^{b}(y-x)^{\alpha-1} f(y) d y,
$$

respectively, where $(-1)^{-\alpha}=\mathrm{e}^{-i \pi \alpha}$ and $\Gamma(\alpha)=\int_{0}^{\infty} r^{\alpha-1} \mathrm{e}^{-r} d r$ denotes the usual Euler function.

If $f \in I_{a+}^{\alpha}\left(L^{p}\right)$ (resp. $f \in I_{b-}^{\alpha}\left(L^{p}\right)$ ) and $\alpha \in(0,1)$, then for almost all $x \in(a, b)$, the left-sided and right-sided Riemann-Liouville derivative of $f$ of order $\alpha$ are defined by

$$
D_{a+}^{\alpha} f(x)=\frac{1}{\Gamma(1-\alpha)}\left(\frac{f(x)}{(x-a)^{\alpha}}+\alpha \int_{a}^{x} \frac{f(x)-f(y)}{(x-y)^{\alpha+1}} d y\right)
$$

and

$$
D_{b-}^{\alpha} f(x)=\frac{1}{\Gamma(1-\alpha)}\left(\frac{f(x)}{(b-x)^{\alpha}}+\alpha \int_{x}^{b} \frac{f(x)-f(y)}{(y-x)^{\alpha+1}} d y\right)
$$

respectively, where $a \leq x \leq b$.

If $\mu \in(0,1)$ and $T \in(0, \infty)$, we note $\mathcal{C}^{\mu}([0, T])$ the set of functions $g:[0, T] \rightarrow$ $\mathbb{R}$ such that

$$
\sup _{0 \leq s<t \leq T} \frac{|g(t)-g(s)|}{|t-s|^{\mu}}<+\infty
$$

If there is no ambiguity, we prefer note $\mathcal{C}^{\mu}$ instead of $\mathcal{C}^{\mu}([0, T])$. The set $\mathcal{C}^{\mu}$ is a Banach space when it is endowed with the following norm:

$$
|g|_{\infty, \mu}:=\sup _{0 \leq t \leq T}|g(t)|+\sup _{0 \leq s<t \leq T} \frac{|g(t)-g(s)|}{|t-s|^{\mu}} .
$$

We also set, for $a, b \in[0, T]$ and $g \in \mathrm{C}^{\mu}$ :

$$
|g|_{a, b, \mu}=\sup _{a \leq s<t \leq b} \frac{|g(t)-g(s)|}{|t-s|^{\mu}} .
$$

and

$$
|g|_{a, b, \infty}=\sup _{a \leq t \leq b}|g(t)| .
$$

When $a=0$ and $b=T$ we simply note $|g|_{\mu}$ and $|g|_{\infty}$ instead of $|g|_{0, T, \mu}$ and $|g|_{0, T, \infty}$ respectively.

Let $f: \mathbb{R} \rightarrow \mathbb{R} \in \mathrm{C}^{\lambda}$ and $g: \mathbb{R} \rightarrow \mathbb{R} \in \mathrm{C}^{\mu}$ with $\lambda, \mu \in(0,1)$ such that $\lambda+\mu>1$. Then, for any $a, b \in[0, T]$, the Young integral [17] $\int_{a}^{b} f d g$ exists and we can express it in terms of fractional derivatives (see [19]): for any $\alpha \in(1-\mu, \lambda$ ), we have

$$
\int_{a}^{b} f d g=(-1)^{\alpha} \int_{a}^{b} D_{a+}^{\alpha} f(x) D_{b-}^{1-\alpha} g_{b-}(x) d x
$$

where $g_{b-}(x)=g(x)-g(b)$. 


\section{First case: when $\sigma$ does not depend of $u$}

In the sequel, we fix $x_{0} \in \mathbb{R}, \beta \in(1 / 2,1), T \in(0, \infty)$ and $g \in \mathcal{C}^{\beta}=\mathcal{C}^{\beta}([0, T])$. We assume moreover that $\sigma:[0, T] \times \mathbb{R} \rightarrow \mathbb{R}$ is $\mathrm{C}^{1,2}$ with bounded derivatives and that $b:[0, T] \times \mathbb{R}^{2} \rightarrow \mathbb{R}$ is bounded and global Lipschitz, uniformly in $x \in \mathbb{R}$ with respect to $(t, u) \in[0, T] \times \mathbb{R}$.

Theorem 1 For any measurable control $u:[0, T] \rightarrow \mathbb{R}$, the integral equation

$$
x_{t}^{u}=x_{0}^{u}+\int_{0}^{t} \sigma\left(r, x_{r}^{u}\right) d g_{r}+\int_{0}^{t} b\left(r, x_{r}^{u}, u_{r}\right) d r, \quad t \in[0, T]
$$

admits a unique solution $x^{u} \in \mathcal{C}^{0}([0, T])$.

\section{Proof of Theorem 1 .}

- We first prove Theorem 1 in the autonomous case, that is when $\sigma(t, x)=\sigma(x)$ and $b(t, x, u)=b(x, u)$. In other words, we consider

$$
x_{t}^{u}=x_{0}^{u}+\int_{0}^{t} \sigma\left(x_{r}^{u}\right) d g_{r}+\int_{0}^{t} b\left(x_{r}^{u}, u_{r}\right) d r, \quad t \in[0, T]
$$

instead of (6). At this level, we need a preliminary lemma:

Lemma 1 Assume that $h:[0, T] \times \mathbb{R}^{3} \rightarrow \mathbb{R}$ is such that, for any $R>0$, there exists $c_{R}>0$ verifying

$\forall(r, g, u, y, z) \in[0, T] \times[-R, R] \times \mathbb{R}^{3}: \quad|h(r, g, u, y)-h(r, g, u, z)| \leq c_{R}|y-z|$,

and that $u:[0, T] \rightarrow \mathbb{R}$ is a measurable function. Then the integral equation

$$
y_{t}=y_{0}+\int_{0}^{t} h\left(r, g_{r}, u_{r}, y_{r}\right) d r, \quad t \in[0, T]
$$

admits a unique solution $y \in \mathrm{C}^{0}([0, T])$.

Proof of Lemma 1. We only sketch the proof, the arguments used being classical.

Existence. Let us define $\left(y^{n}\right)$ recursively by $y^{0}(t) \equiv y_{0}$ and

$$
y^{n+1}(t)=y_{0}+\int_{0}^{t} h\left(r, g(r), u(r), y^{n}(r)\right) d r, \quad t \in[0, T] .
$$


Since $g$ is continuous, there exists $R>0$ such that $g([0, T]) \subset[-R, R]$. Thus, using the hypothesis made on $h$, it is classical to prove that $\left|y^{n+1}-y^{n}\right|_{\infty} \leq \frac{c_{R}{ }^{n}}{n !}$. In particular, the sequence $\left(y^{n}\right)$ is Cauchy and the limit $y$ is a solution to (9). Uniqueness. Let $y$ and $z$ be two solutions of (9). Then, for any $t \in[0, T]$, we easily have

$$
|y-z|_{\infty,[0, t]} \leq c_{R} \int_{0}^{t}|y-z|_{\infty,[0, r]} d r
$$

and we can conclude that $y=z$ using Gronwall's lemma.

We now apply the Doss-Sussmann's method in order to finish the proof of Theorem 1 in the autonomous case. First, we denote by $\phi$ the unique solution to

$$
\frac{\partial \phi}{\partial g}(g, y)=\sigma \circ \phi(g, y), \forall g, y \in \mathbb{R} \text { and } \phi(0, y)=y, \forall y \in \mathbb{R} .
$$

The hypothesis made on $\sigma$ ensures that $\phi$ is well-defined. We also have, for $g, y \in \mathbb{R}:$

$$
\frac{\partial \phi}{\partial y}(g, y)=\exp \left(\int_{0}^{g} \sigma^{\prime}(\phi(h, y)) d h\right)
$$

Define $f: \mathbb{R}^{3} \rightarrow \mathbb{R}$ by

$$
f(g, u, y)=\frac{b(\phi(g, y), u)}{\frac{\partial \phi}{\partial y}(g, y)}=b(\phi(g, y), u) \exp \left(-\int_{0}^{g} \sigma^{\prime}(\phi(\ell, y)) d \ell\right) .
$$

The hypothesis made on $b$ and $\sigma$ ensures that $h:[0, T] \times \mathbb{R}^{3} \rightarrow \mathbb{R}$ defined by $h(r, g, u, y)=f(g, u, y)$ verifies (8). Thus, there exist a unique $y$ solution to (9). Using the change of variable formula, it is now immediate to prove that $x_{t}^{u}=\phi\left(g_{t}, y_{t}\right)$ is a solution to (7). For the uniqueness, it suffices to adapt to our context the proof contained in [2], page 103.

- The general case being similar with the previous case, we only sketch the proof.

Here, we have to consider $\phi$ given by

$$
\frac{\partial \phi}{\partial g}(r, g, y)=\sigma(r, \phi(r, g, y)), \forall(r, g, y) \in[0, T] \times \mathbb{R}^{2}
$$

with initial conditions

$$
\phi(r, 0, y)=y, \forall(r, y) \in[0, T] \times \mathbb{R}
$$


instead of (10). Moreover, $y:[0, T] \rightarrow \mathbb{R}$ is, in the case, defined as the unique solution to (9) with $h$ given by

$$
h(r, g, u, y)=\frac{b(r, \phi(r, g, y), u)-\frac{\partial \phi}{\partial r}(r, g, y)}{\frac{\partial \phi}{\partial y}(r, g, y)},
$$

see also [2], page 116. Finally, the unique solution to (6) is given by

$$
x_{t}^{u}=\phi\left(t, g_{t}, y_{t}\right)
$$

In order to make use of a compactification method, it is necessary to enlarge the set of controls by considering relaxed controls.

Definition 1 A relaxed control is a measure $q$ over $U \times[0, T]$ such that the projection of $q$ on $[0, T]$ is the Lebesgue measure. We denote by $\mathcal{V}$ the set of relaxed controls.

A relaxed control $q$ can be decomposed with a measurable kernel: $q(d a, d t)=$ $q_{t}(d a) d t$ where $t \mapsto q_{t}$ is a measurable function from $\mathbb{R}^{+}$to the set of probability measures on $U$. There is a natural embedding of (non-relaxed) controls in the set of relaxed controls: $q$ is a non-relaxed control if at each time $t, q_{t}$ concentrates on a single point $u_{t}$. In other words, we assimilate the control $\left(u_{t}\right)_{t \in[0, T]}$ with the relaxed control $\delta_{u_{t}} d t$ where $\delta_{x}$ denotes the Dirac measure at $x$. We denote by $\mathcal{V}^{\prime}$ the set of non-relaxed controls.

The main result that we shall need is the immediate following consequence of the vague topology.

Proposition 1 Suppose $U$ is a compact subset of $\mathbb{R}^{n}$. The set $\mathcal{V}$ of relaxed controls equipped with the vague topology is compact.

From now on, we shall suppose that the set $U$ is compact. A solution to equation (6) associated to a relaxed control $q$ is obtained in the following extension of Theorem 1 .

Theorem 2 Let $q \in \mathcal{V}$ be a relaxed control. There exists a unique solution $x^{q} \in$ $C^{0}([0, T])$ of the equation

$$
x_{t}^{q}=x_{0}^{q}+\int_{0}^{t} \sigma\left(r, x_{r}^{q}\right) d g_{r}+\int_{0}^{t} \int_{U} b\left(r, x_{r}^{q}, a\right) q_{r}(d a) d r .
$$

Moreover, $q \mapsto x^{q}$ is continuous from $\mathcal{V}$ to $C^{0}([0, T])$. 
Proof. Denote by $\phi$ the unique solution to (11). Set

$$
h(r, g, q, y)=\frac{\int_{U} b(r, \phi(r, g, y), a) q_{r}(d a)-\frac{\partial \phi}{\partial r}(r, g, y)}{\frac{\partial \phi}{\partial y}(r, g, y)} .
$$

Clearly, due to the hypotheses on $b$ and $\sigma, \forall(r, g, q, y, z) \in[0, T] \times[-R, R] \times \mathcal{V} \times \mathbb{R} \times \mathbb{R}$,

$$
|h(r, g, q, y)-h(r, g, q, z)| \leq c_{R}|y-z| \text {. }
$$

Therefore, the integral equation (9) admits a unique solution $y \in C^{0}([0, T])$, see Lemma 1. Then, one may check that $x_{t}^{q}=\phi\left(t, g_{t}, y_{t}\right)$ is a solution to (12). Uniqueness is obtained as before.

Suppose now that $q^{n}$ is a sequence in $\mathcal{V}$, converging to $q \in \mathcal{V}$ and let $y^{n}$ be the solution of (9) associated to $h=h\left(r, g, q^{n}, y\right)$ given by (13). Using hypotheses on $b$, we now prove that $y^{n}$ converges to $y$ in $C^{0}([0, T])$. Indeed,

$$
\begin{aligned}
& \left|y_{t}-y_{t}^{n}\right|=\left|\int_{0}^{t}\left[h\left(s, g_{s}, q, y_{s}\right)-h\left(s, g_{s}, q^{n}, y_{s}^{n}\right)\right] d s\right| \\
& \leq\left|\int_{0}^{t} h\left(s, g_{s}, q, y_{s}\right) d s-\int_{0}^{t} h\left(s, g_{s}, q^{n}, y_{s}\right) d s\right| \\
& +\left|\int_{0}^{t} h\left(s, g_{s}, q^{n}, y_{s}\right) d s-\int_{0}^{t} h\left(s, g_{s}, q^{n}, y_{s}^{n}\right) d s\right| \\
& \leq\left|\int_{0}^{t} \int_{U} b\left(s, \phi\left(s, g_{s}, y_{s}\right), a\right) q_{s}^{n}(d a) d s-\int_{0}^{t} \int_{U} b\left(s, \phi\left(s, g_{s}, y_{s}\right), a\right) q_{s}(d a) d s\right| \\
& +c_{R} \int_{0}^{t}\left|y_{s}-y_{s}^{n}\right| d s \text {. }
\end{aligned}
$$

In the last expression, the first term tends to 0 due to the vague convergence of $q_{s}^{n}(d a) d s$ to $q_{s}(d a) d s$, and continuity and boundedness hypotheses on $b$. It results therefore from Gronwall's lemma that $\left|y-y^{n}\right|_{\infty}$ tends to 0. Finally, as the solution $x^{q}$ (resp. $x^{q^{n}}$ ) of (12) associated to $q$ (resp. $q^{n}$ ) is given by $x_{t}=\phi\left(t, g_{t}, y_{t}\right)$ (resp. $\left.x_{t}^{n}=\phi\left(t, g_{t}, y_{t}^{n}\right)\right)$, one easily deduces that $\left|x-x^{n}\right|_{\infty}$ tends to 0 .

Consider now a cost in integral form: for $u_{t}$ a given control taking values in $U$, we set

$$
J(u)=\int_{0}^{T} \ell\left(r, x_{r}^{u}, u_{r}\right) d r
$$

where $\ell$ is a bounded continuous function on $(r, x, u)$. The definition can be immediately extended to the case of relaxed controls: if $q$ is a relaxed control from $\mathcal{V}$,

$$
J(q)=\int_{0}^{T} \int_{U} \ell\left(r, x_{r}^{q}, a\right) q_{r}(d a) d r .
$$

Using the continuity property of Theorem 2, and the hypotheses on $\ell$, one obtains the following Proposition. 
Proposition 2 Under the hypotheses introduced in the previous paragraph, the application $q \mapsto J(q)$ is continuous on $\mathcal{V}$.

The set $\mathcal{V}$ being compact, one immediately deduces the following existence result.

Corollary 1 Under the prevailing hypotheses, there exists $q^{*} \in \mathcal{V}$ such that

$$
J\left(q^{*}\right)=\inf _{q \in \mathcal{V}} J(q) .
$$

We conclude the present section by proving that one has not enlarged too much the control problem by considering relaxed controls. More precisely, we now prove that the optimal cost (i.e. the infemum of the cost functional) over relaxed and non-relaxed controls is the same. This result is obtained as in the deterministic case by means of approximation of relaxed controls by step constant relaxed controls, and then by non-relaxed controls via the so-called chattering lemma, a method originally introduced in [6]. We here only sketch these two steps.

First step $: q \in \mathcal{V}$ is approximated by relaxed controls of the form

$$
\sum_{j=0}^{N-1} \sum_{i=1}^{k} q_{i}^{j} \delta_{a_{i}}(d a) \mathbf{1}_{\left[t_{j}, t_{j+1}[\right.}
$$

where $0=t_{0}<t_{1}<\cdots<t_{N}=T, a_{1}, \ldots, a_{k}$ are elements in $U$, and for each $j=0, \ldots, N-1, q_{1}^{j}, \ldots, q_{k}^{j}$ are non-negative real numbers such that $\sum_{i=1}^{k} q_{i}^{j}=1$. This is a straightforward consequence of approximation of the measurable function $t \mapsto q_{t}$ by a step function and of approximation of a probability measure $\mu$ on $U$ by point measures of the form $\sum_{i=1}^{m} \mu_{i} \delta_{a_{i}}$.

Second step: Recall the chattering lemma (see [6], Theorem 1)

Proposition 3 Let $a_{1}, \ldots, a_{k}$ be in $U$ and $q_{1}, \ldots, q_{k}$ be non-negative real numbers such that $\sum_{i=1}^{k} q_{i}=1$. Let $f$ be a bounded continuous function from $[s, t] \times U$ to $\mathbb{R}$. Then, for $\varepsilon>0$ given, there exists a measurable partition $V_{1}, \ldots, V_{k}$ of $[s, t]$ such that

$$
\left|\int_{s}^{t} \sum_{i=1}^{k} q_{i} f\left(r, a_{i}\right) d r-\sum_{i=1}^{k} \int_{V_{i}} f\left(r, a_{i}\right) d r\right|<\varepsilon
$$


In other words, the step-relaxed control $\left(\sum_{i=1}^{k} q_{i} \delta_{a_{i}}(d a)\right) d r$ is approximated by the non-relaxed control $\sum_{i=1}^{k} \mathbf{1}_{V_{i}}(r) \delta_{a_{i}}(d a) d r$. Therefore, for any $q \in \mathcal{V}$, there exists a sequence of (non-relaxed) controls $q^{n}$ which converges to $q$. As, obviously, the infemum of $J$ on $\mathcal{V}$ is smaller than the infemum on $\mathcal{V}^{\prime}$, using the continuity of $J$, we obtain the following comparison result.

Proposition 4 Under the hypotheses of the present section, $\inf _{q \in \mathcal{V}} J(q)=\inf _{q \in \mathcal{V}^{\prime}} J(q)$.

\section{Second case: when $\sigma$ depends of $u$}

As already mentioned in the introduction, the case when $u$ enters the coefficient of $d g_{t}$ seems to be much more complicated as we do not have a reasonable way for integrating functions less regular than Hölderian. Therefore we shall need to restrict very strongly our admissible controls set.

In the sequel, we fix $x_{0} \in \mathbb{R}, \beta \in(1 / 2,1), T \in(0, \infty), g \in \mathcal{C}^{\beta}=\mathcal{C}^{\beta}([0, T])$, $\sigma:[0, T] \times \mathbb{R}^{2} \rightarrow \mathbb{R} \in \mathrm{C}^{1,2,2}$ with bounded derivatives and $b:[0, T] \times \mathbb{R}^{2} \rightarrow \mathbb{R}$ global Lipschitz continuous.

Theorem 3 For any control $u \in \mathcal{C}^{\mu}$ with $1-\beta<\mu \leq \beta$, the integral equation

$$
x_{t}^{u}=x_{0}^{u}+\int_{0}^{t} \sigma\left(r, x_{r}^{u}, u_{r}\right) d g_{r}+\int_{0}^{t} b\left(r, x_{r}^{u}, u_{r}\right) d r, \quad t \in[0, T]
$$

admits a unique solution $x^{u} \in \mathcal{C}^{\mu}$.

Proof of Theorem 3. It suffices to adapt the proof of Ruzmaikina [16 to our context, i.e. to hold account of the control $u$. There is not new difficulties. See also Nualart and Răsçanu [15].

Theorem 4 If $\mathcal{U}$ is a set of functions which is bounded in a certain $\mathcal{C}^{\mu}$ with $\mu \in$ $(1-\beta, \beta]$ and if $J: \mathcal{U} \rightarrow \mathbb{R}$ is continuous for $|\cdot|_{\infty, \mu^{\prime}}$ for a certain $\mu^{\prime} \in(1-\beta, \mu)$ then the following control problem can be solved:

there exists $u^{*} \in \mathcal{U}$ realizing $\inf _{u \in \mathcal{U}} J(u)$.

Proof of Theorem 4. It is a direct consequence of Lemma 2 below. 
Lemma 2 If $\mathcal{U}$ is a set of functions which is bounded in a certain $\mathcal{C}^{\mu}$ with $\mu \in$ $(1-\beta, \beta]$ then the set of all couples $\left(u, x^{u}\right) \in \mathcal{U} \times \mathcal{C}^{\mu}$ is relatively compact in $\mathcal{C}^{\mu^{\prime}} \times \mathcal{C}^{\mu^{\prime}}$ for any $\mu^{\prime} \in(1-\beta, \mu)$.

Proof of Lemma 2. According to Lamperti [12], we know that $\mathcal{U}$ is relatively compact in $\mathcal{C}^{\mu^{\prime}}$. Lemma 3 below allows then to conclude.

Lemma 3 For any $\mu \in(1-\beta, \beta], T^{\mu}: \mathcal{C}^{\mu} \rightarrow \mathcal{C}^{\mu}$ defined by $T^{\mu}(u)=x^{u}$ is a continuous operator.

Proof of Lemma 3. We adapt the proof of Theorem 3.2 in $\mathrm{Hu}$ and Nualart [7]. For simplicity, we assume that $\sigma(r, x, u)=\sigma(x, u)$ and $b(r, x, u)=b(x, u)$, the proof of the general case being similar. Moreover positive constants, depending only on $b$, $\sigma$, their derivatives, $x_{0}$ and $g$, will be denoted by $k$, regardless of their value. Let $\left(u^{n}\right) \subset \mathcal{C}^{\beta}$ be such that $u^{n} \rightarrow u$. Fix $s, t \in[0, T]$ and let $\alpha \in(1-\beta, \mu)$. We can write, using (5):

$$
\begin{aligned}
& \left|x_{t}^{u}-x_{t}^{u_{n}}-x_{s}^{u}+x_{s}^{u_{n}}\right| \\
= & \left|\int_{s}^{t}\left[\sigma\left(x_{r}^{u}, u_{r}\right)-\sigma\left(x_{r}^{u_{n}}, u_{r}^{n}\right)\right] d g_{r}+\int_{s}^{t}\left[b\left(x_{r}^{u}, u_{r}\right)-b\left(x_{r}^{u_{n}}, u_{r}^{n}\right)\right] d r\right| \\
\leq & \int_{s}^{t}\left|D_{s+}^{\alpha}\left[\sigma\left(x_{r}^{u}, u_{r}\right)-\sigma\left(x_{r}^{u_{n}}, u_{r}^{n}\right)\right]\right| \cdot\left|D_{t-}^{1-\alpha} g_{t-}(r)\right| d r+\int_{s}^{t}\left|b\left(x_{r}^{u}, u_{r}\right)-b\left(x_{r}^{u_{n}}, u_{r}^{n}\right)\right| d r .
\end{aligned}
$$

Using (4), it is easy, on one hand, to show that

$$
\left|D_{t-}^{1-\alpha} g_{t-}(r)\right| \leq k|g|_{\beta}|t-r|^{\alpha+\beta-1} .
$$

On the other hand, we have, using (3):

$$
\begin{aligned}
& \left|D_{s+}^{\alpha}\left[\sigma\left(x_{r}^{u}, u_{r}\right)-\sigma\left(x_{r}^{u_{n}}, u_{r}^{n}\right)\right]\right| \\
\leq & \left|\sigma^{\prime}\right|_{\infty}\left(\left|x^{u}-x^{u_{n}}\right|_{s, t, \infty}+\left|u-u^{n}\right|_{s, t, \infty}\right)(r-s)^{-\alpha} \\
+ & \left|\sigma^{\prime}\right|_{\infty}\left(\left|x^{u}-x^{u_{n}}\right|_{s, t, \mu}+\left|u-u^{n}\right|_{s, t, \mu}\right)(r-s)^{\mu-\alpha} \\
+ & \left|\sigma^{\prime \prime}\right|_{\infty}\left(\left|x^{u}-x^{u_{n}}\right|_{s, t, \infty}+\left|u-u^{n}\right|_{s, t, \infty}\right)\left(\left|x^{u_{n}}\right|_{s, t, \mu}+\left|u^{n}\right|_{s, t, \mu}\right)(r-s)^{\mu-\alpha} .
\end{aligned}
$$

We deduce that

$$
\begin{aligned}
& \left|x^{u}-x^{u_{n}}\right|_{s, t, \mu} \\
\leq & k\left[\left(\left|x^{u}-x^{u_{n}}\right|_{s, t, \infty}+\left|u-u^{n}\right|_{s, t, \infty}\right)(t-s)^{\beta-\mu}+\left(\left|x^{u}-x^{u_{n}}\right|_{s, t, \mu}+\left|u-u^{n}\right|_{s, t, \mu}\right)(t-s)^{\beta}\right. \\
+ & \left.\left(\left|x^{u}-x^{u_{n}}\right|_{s, t, \infty}+\left|u-u^{n}\right|_{s, t, \infty}\right)\left(\left|x^{u_{n}}\right|_{s, t, \mu}+\left|u^{n}\right|_{s, t, \mu}\right)(t-s)^{\beta}\right]^{\beta}
\end{aligned}
$$

and, by rearranging:

$$
\begin{aligned}
& \left|x^{u}-x^{u_{n}}\right|_{s, t, \mu} \\
\leq & k\left(1-k(t-s)^{\beta}\right)^{-1}\left[\left|x^{u}-x^{u_{n}}\right|_{s, t, \infty}+\left|u-u^{n}\right|_{s, t, \infty}+\left|u-u^{n}\right|_{s, t, \mu}(t-s)^{\beta}\right. \\
+ & \left.\left(\left|x^{u}-x^{u_{n}}\right|_{s, t, \infty}+\left|u-u^{n}\right|_{s, t, \infty}\right)\left(\left|x^{u_{n}}\right|_{s, t, \mu}+\left|u^{n}\right|_{s, t, \mu}\right)(t-s)^{\beta}\right] .
\end{aligned}
$$


Then, if we set $\Delta=t-s$ :

$$
\begin{aligned}
& \left|x^{u}-x^{u_{n}}\right|_{s, t, \infty} \\
\leq & \left|x_{s}^{u}-x_{s}^{u_{n}}\right|+\left|x^{u}-x^{u_{n}}\right|_{s, t, \mu} \Delta^{\beta} \\
\leq & \left|x_{s}^{u}-x_{s}^{u_{n}}\right|+k\left(1-k \Delta^{\beta}\right)^{-1} \Delta^{\beta}\left[\left|x^{u}-x^{u_{n}}\right|_{s, t, \infty}+\left|u-u^{n}\right|_{s, t, \infty}\right. \\
+ & \left.\left|u-u^{n}\right|_{s, t, \mu} \Delta^{\beta}+\left(\left|x^{u}-x^{u_{n}}\right|_{s, t, \infty}+\left|u-u^{n}\right|_{s, t, \infty}\right)\left(\left|x^{u_{n}}\right|_{s, t, \mu}+\left|u^{n}\right|_{s, t, \mu}\right) \Delta^{\beta}\right] .
\end{aligned}
$$

By rearranging, we obtain

$$
\begin{aligned}
& \left|x^{u}-x^{u_{n}}\right|_{s, t, \infty} \leq\left[1-k\left(1-k \Delta^{\beta}\right)^{-1} \Delta^{\beta}\left(1+\left(\left|x^{u_{n}}\right|_{s, t, \mu}+\left|u^{n}\right|_{s, t, \mu}\right) \Delta^{\beta}\right)\right]^{-1} \\
& \times\left(\left|x_{s}^{u}-x_{s}^{u_{n}}\right|+k\left(1-k \Delta^{\beta}\right)^{-1} \Delta^{\beta}\left[\left|u-u^{n}\right|_{\infty, \mu}+\left|u-u^{n}\right|_{\infty}\left(\left|x^{u_{n}}\right|_{\mu}+\left|u^{n}\right|_{\mu}\right)\right]\right) .
\end{aligned}
$$

We can finish as in [7] to obtain that $\left|x^{u}-x^{u_{n}}\right|_{\infty} \rightarrow 0$ as $n \rightarrow \infty$. Using finally (15), we obtain that $\left|x^{u}-x^{u_{n}}\right|_{\infty, \mu} \rightarrow 0$ as $n \rightarrow \infty$, that is $x^{u_{n}} \rightarrow x^{u}$ in $\mathcal{C}^{\mu}$. In other words, $T^{\mu}$ is a continuous operator from $\mathcal{C}^{\mu}$ to himself.

An example of a cost $J$ satisfying conditions of Theorem 4 is

$$
J(u)=\int_{0}^{T} \ell\left(r, x_{r}^{u}, u_{r}\right) d r
$$

with $\ell:[0, T] \times \mathbb{R}^{2} \rightarrow \mathbb{R}$ verifying

$$
\forall(r, x, y, u, v) \in[0, T] \times \mathbb{R}^{4}, \quad|\ell(r, x, u)-\ell(r, y, v)| \leq \operatorname{cst}(|x-y|+|u-v|) .
$$

\section{References}

[1] Coutin, L. (2006), An introduction to (stochastic) calculus with respect to fractional Brownian motion, To appear in Séminaires de Probabilités (2006).

[2] Doss, H., Liens entre équations différentielles stochastiques et ordinaires, Ann. Inst. Henri Poincaré 13, no. 2 (1977), 99-125.

[3] Duncan, T.E., Hu, Y. and Pasik-Duncan, B., Stochastic calculus for fractional Brownian motion I. Theory, SIAM J. Cont. Opt. 38 (2000), 582-612.

[4] El Karoui, N., Huu Nguyen, D. and Jeanblanc-Picqué, M., Compactification Methods in the Control of Degenerate Diffusions : Existence of an Optimal Control, Stochastics 20 (1987), 169-219.

[5] Fleming, W.H., Generalized solutions in optimal stochastic control, Differential Games and Control Theory, Kingston Conference 2, Lecture Notes in Pure and Applied Math. 30 (1978), Dekker. 
[6] Ghouila-Houri, A., Sur la généralisation de la notion de commande d'un système guidable, RIRO 4 (1967), 7-32.

[7] Hu, Y. and Nualart, D., Differential equations driven by Hölder continuous functions of order greater than $1 / 2$, Prepublication available on http://arxiv.org/pdf/math.PR/0601628 (2006).

[8] Hu, Y. and Nualart, D., Rough path analysis via fractional calculus, Prepublication available on http://arxiv.org/pdf/math.PR/0602050 (2006).

[9] Hu, Y., Öksendal, B. and Sulem, A., Optimal consumption and portfolio in a Black-Scholes market driven by fractional Brownian motion, Inf. Dim. Anal. Quant. Prob. 6 (2003), 519-536.

[10] Hu, Y. and Zhou X.Y., Stochastic control for linear systems driven by fractional noises, SIAM J. Cont. Opt. 43, no. 6 (2005), 2245-2277.

[11] Kleptsyna, M.L., Le Breton, A. and Viot, M., About the linear quadratic regulator problem under a fractional Brownian perturbation, ESAIM Prob. Stat. 7 (2003), 161-170.

[12] Lamperti, J., On convergence of stochastic processes, American Mathemat. Society Transact. 104 (1962), 430-435.

[13] Lyons, T.J., Differential equations driven by rough signals, Rev. Mat. Iberoamericana 14, no. 2 (1998), 215-310.

[14] Nourdin, I., Simon, T., Correcting Newton-Côtes integrals by Lévy areas, Prepublication available on http://arxiv.org/pdf/math.PR/0601544 (2006).

[15] Nualart, D., Răsçanu, A., Differential equations driven by fractional Brownian motion, Collect. Math. 53, no. 1 (2002), 55-81.

[16] Ruzmaikina, A.A., Stieltjes integrals of Hlder continuous functions with applications to fractional Brownian motion, J. Statist. Phys. 100, no. 5-6 (2000), 1049-1069.

[17] Young, L.C., An inequality of the Hölder type connected with Stieltjes integration, Acta Math. 67 (1936), 251-282.

[18] Young L.C., Lectures on the Calculus of Variations and Optimal Control Theory. W. B. Saunders Co., Philadelphia-London-Toronto, Ont., 331 pages, 1969.

[19] Zähle, M., Integration with respect to fractal functions and stochastic calculus I, Probab. Theory Relat. Fields 111 (1998), 333-374. 